\title{
Experimental Assessment of a Multi-Variable Control Strategy of a Micro-Cogeneration Solar-ORC Plant for Domestic Application
}

\author{
Diego Vittorini ${ }^{1,}$, , Fabio Fatigati ${ }^{1}$, Davide Di Battista ${ }^{1}$, Marco Di Bartolomeo ${ }^{1}$, Roberto Carapellucci ${ }^{1}$ \\ ${ }^{1}$ University of L'Aquila, Department of Industrial and Information Engineering and Economics, 67100, L'Aquila, Italy
}

\begin{abstract}
Suitability to off-design operation, applicability to combined thermal and electrical generation in a wide range of low temperatures and pressures and compliance with safety and environmental limitations qualify small-scale Organic Rankine Cycle plants as a viable option for combined heat and power generation in the residential sector. As the plants scale down, the electric and thermal output maximization has to account for issues, spanning from high pump power absorption, compared to the electric output of the plant, to intrinsically low plant permeability induced by the expander, to the intermittent availability of thermal power, affected by the heat demand for domestic hot water (DHW) production. The present paper accounts for a flat-plate solar thermal collector array, bottomed by an ORC unit featuring a sliding vane expander and pump and flat-plate heat exchangers. A high-temperature buffer vessel stores artificially heated water electric heaters, simulating the solar collector - and feeds either the hot water line for domestic use or the ORC evaporator, depending on the instantaneous demand (i.e., domestic hot water or electric power), the temperature conditions inside the tank and the stored mass availability. A low-temperature receiver acts like the heat sink of the ORC unit and harvests the residual thermal power, downstream the expander: a dedicated control, modelled to properly modulate the mass addition/subtraction to this storage unit allows to restore the operating points of the cycle and to limit the incidence of off-design operation, via real-time adjustment of the cycle operating parameters. Indeed, the possibility of continuous ORC generation depends on (i) the nature of the demand and (ii) the amount of hot water withdrawn from the high-temperature buffer vessel. The time-to-temperature for the mass stored inside the buffer affects the amount of ORC unit activations and eventually the maximum attainable generation of electric energy. The plant energy performance is experimentally assessed, and various characteristic operating points are mapped, based on test runs carried out on a real-scale ORC pilot unit.
\end{abstract}

\section{Introduction}

Micro-ORC units, fed by thermal energy from solar thermal collectors, have an enormous potential for distributed electricity generation and contemporary coverage of both thermal and electrical demand in the Residential sector [1-3]. Their suitability to low-temperature thermal sources makes the concentration of solar radiation unnecessary - saving investment costs and plant complexity [4-8] - and reduces both operating temperatures and pressures, leading to better compliance with fail-safe, durability and lower maintenance requirements applications in the Residential sector must meet [9-12].

The variability, on a seasonal and hourly basis, of the thermal demand for domestic hot water (DHW) production and heating purposes, calls for the integration to the plant of a vessel for thermal energy storage (TES), to serve the dual purpose of (i) decoupling the thermal availability at the evaporator from any environment-induced disturbance [13-16] and (ii) harvesting thermal energy during no DHW-withdrawals to feed it to the power section for electricity generation. Plus, the proper filling-emptying dynamics of the storage unit is the key to both (i) optimum ORC unit sizing and (ii) prevention of ORC off-design operation, resulting in stabler operation, higher energy performance for plant components and larger net power generation $[17,18]$.

Various TES configurations can be retrieved from the scientific literature, where both experimental and model-based approaches allow the proper characterization of (i) single- and two-tanks systems [19, 20], (ii) heat-transfer fluids ranging from pure fluids to mixtures - molten-salts and eutectic - and phase change materials [21,22] and (iii) direct and indirect storage configurations $[23,24]$. Nonetheless, they all refer to CSP-bottoming ORC-units, for which the higher investment

*Corresponding Author: diego.vittorini@univaq.it 
and maintenance costs are compensated by the larger potential energy output from the ORC-plant. Plus, the more the plant drifts to small scales, the more important its ability becomes, to provide a fast response to (i) demand fluctuations and (ii) daily thermal and electrical profile peaks, without significant penalty on the energy performance.

Consequently, the residual margin for plant enhancement should be exploited through the definition of a control strategy, able to properly cope with (i) the need for plant downsizing (e.g., reduced heat exchange surfaces, reduced mass flowrates of working fluid), (ii) the limitations on the applicable fluid type [25-27] and (iii) the constraints on admissible operating temperatures and pressures [28-30]. Both the low characteristic mass flowrates of working fluid and the variability of the thermal energy at the evaporator call for dedicated design of rotary equipment [31-33].

More specifically, the expander design has to account for the possibility of a two-phase expansion, with a variable superheating degree and proper operation in a wide RPM range. In addition, the need for mass flowrate modulation via proper selection of the pump speed, calls for a pump designed for reduced energy absorption within the whole operating RPM range [33]. As a matter of fact, mass flowrate, cycle pressures (namely, the pressure at expander inlet) and expander speed are inter-related, particularly in volumetric machines, where the processing of a variable mass flowrate is associated with speed modulation: to keep the cycle operating pressures (namely, the pressure at expander inlet) unaffected in presence of an increased mass flowrate, a higher expander RPM is needed, to allow a quicker process of vane fillingemptying. Nonetheless, this ends up affecting lubrication and friction losses and eventually the plant energy output [33].

In the present paper, the results of an extensive experimental campaign on a prototype ORC-unit, are presented: based on a preliminary parametric study [34] to account for off-design operation of the plant, the energy performance of the system is assessed. Electrically heated water reproduces the thermal availability from flat plate solar thermal collectors at a $250 \mathrm{~L}$ tank. The thermal energy fed to the ORC evaporator is controlled to reproduce the combined effect of variable solar availability, collector thermal dynamics and absorption for DHW production. The speed control at the pump allows the mass flowrate of working fluid (R245fa) modulation. In addition, the expander RPM can be set to different values, hence opening the way to the assessment of the plant hydraulic permeability under real operating conditions.

The real-time monitoring of operating temperatures and pressures allows the reproduction of the thermodynamic cycle associated with the plant, featuring a volumetric pump and a sliding vane expander. Plate and fins heat exchangers complete the base layout. Different set-point temperatures for ORC-activation are considered and result in different $\mathrm{R} 245 \mathrm{fa}$ mass flowrates processed in the power section: for each operating point, the expander speed for maximum plant hydraulic permeability is assessed, along with the effects of sub-optimum lubrication and larger friction losses.

In light of the typically very low characteristic conversion efficiency of ORC-based plants, a large share of the thermal power provided to the organic fluid at the evaporation section is still available at the expander outlet: the system integrates a low-temperature buffer vessel to assist the system in the primary purpose of DHW production and increase the amount of thermal energy potentially available to electric generation.

Both continuous and unsteady operation underwent an in-depth analysis, as well as the benefits associated with different discharge times (i.e. either a flash or a progressive tank discharge) for the storage unit, to maximize, in turn, the electrical and thermal output and to (i) allow the cogeneration unit to operate with little-to-no irradiance, when electricity is typically most required, (ii) allow the instantaneous matching of both electric and thermal demand and (iii) in case of over-production of electric power, to plan the proper management of battery packs or the sale to the grid when it is more valuable, for financial revenue.

\section{Micro-ORC Unit Prototype}

Fig. 1 reports the prototype of the single evaporation pressure, subcritical cycle, under investigation. The ORC-unit features a condenser (a) and an expansion vessel (b), placed downstream the condenser, for pressure oscillations dampening; the fluid mass flowrate, processed by the diaphragm pump (c) is measured by a Coriolis-flowmeter (d) and is fed to the flat plate evaporator (e) where a slight superheating occurs (between $5^{\circ} \mathrm{C}$ and $13^{\circ} \mathrm{C}$ ), with characteristic cycle upper pressures in the 6 bar to 11 bar range; it finally expands within the sliding vane expander (f).

Once the pressure difference upstream and downstream the expander is large enough to provide a driving torque that overcomes inertia and friction, the rotor is dragged into rotation: the role played by the generator is then crucial, as it can be either used in reverse operation (i.e. as an electric motor) to speed-up the expander to the design speed; or it can be connected to the expander shaft and provide the resisting load that keeps the expander RPM to the speed value of choice. The former option has its main advantage of a shorter time-to-regime for the expander and is particularly effective in presence of larger masses to move (i.e. larger mechanical inertia to overcome) and larger thermal inertia of the plant (e.g. larger mass flowrates of the working fluid); in the case at hand, the dedicated design of the expander - assuring reduced mechanical inertia - makes the resisting torque on the expander shaft negligible and easily overcome by the enthalpy content the fluid provides, during thermal transients already, hence, the generator is never employed as an electric motor. 
As a matter of fact, when the goal is to maximize the electricity generation, the option of a self-activated expander should be preferred, as it configures the possibility of extending the operating range of the power unit to the initial transients, otherwise unexploited.

Apart from the satisfying trade-off it assures, between high characteristic efficiencies and mass flowrate variability in low-grade waste-heat recovery, the sliding vane technology is particularly appreciated, since it assures tolerance to dualphase expansions and for smooth operation in off design conditions: the less stringent constraints on the selection of the superheating degree creates conditions for lower losses for irreversibility at the heat exchanger and on a 1-3\% gain on the overall energy efficiency of the system, particularly valuable in small scale systems, usually suffering from a high Back-Work ratio (i.e. ratio between the work for pumping and the work gathered at the expander) [31, 34].

The plant is charged with $6 \mathrm{~kg} \mathrm{R} 245 \mathrm{fa}$ mixed with oil (5\% weight fraction) for sealing and lubrication purpose. Despite the experimental campaign at hand did not account for any regeneration, the ORC-unit layout includes a flat plate heat exchanger (g) to subtract the residual thermal content of the fluid at the expander outlet and exploit the dual advantage of pre-heating the fluid - prior it approaches the evaporator, hence reducing the heat subtraction to DHW purposes - and downscaling the condenser, with smaller heat exchanging surfaces required.

The plant set-points - expander and pump RPM - are input via a control board (h), that also allows the real-time reading of signals from piezoresistive sensors and thermocouples (n) for thermodynamic cycle reconstruction, torquemeter (m) on the expander shaft, to assess the available energy from the expansion, and the mass flowrate values of both R245fa (Coriolis type) and water (magnetic type) at the evaporator hot side and condenser cold side (Table 1).

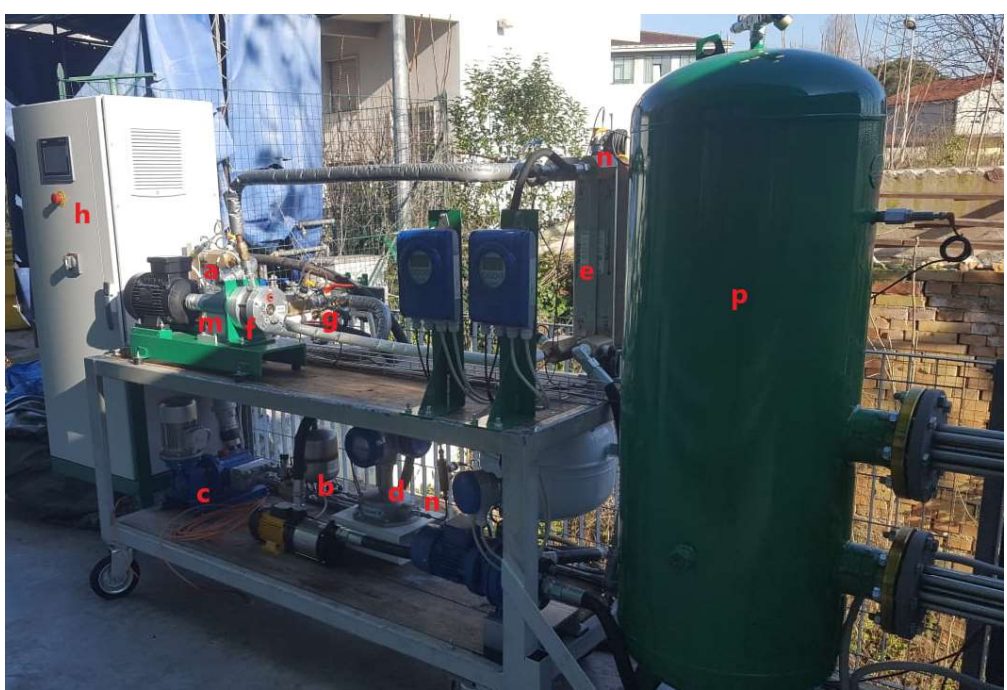

Fig. 1. ORC-plant prototype.

A major breakthrough with respect to more traditional applications, is the expander speed modulation via a dedicated regenerative inverter, that decouples the speed of the expander from the net frequency, hence widening the operating range the investigation can account for and reproducing continuous plant operation, closer to the actual operation mode for the plant. The vessel (p) integrates electric heaters to reproduce the thermal power, the water would receive from the solar thermal collectors during in-field operation: the adduction to ORC-evaporator only occurs when the temperature threshold for ORC-unit activation is reached. 
Table 1. Measurement equipment and sensors accuracy.

\begin{tabular}{ccc}
\hline Variable & Sensor type & Accuracy \\
\hline Temperature & T-type thermocouple & $\pm 0,75{ }^{\circ} \mathrm{C}$ \\
Pressure & Piezoresistive sensor & $\pm 1,5 \%$ full scale \\
Mass flowrate (R245fa) & Coriolis-type sensor & $\pm 0,15 \%$ measured value \\
Mass flowrate (water) & Magnetic-type sensor & $\pm 0,5 \%$ measured value \\
Torque & Torquemeter & $\pm 0,02 \mathrm{Nm}$ \\
Power & Wattmeter & $\pm 0,5 \%$ measured value \\
\hline
\end{tabular}

\section{Experimental Characterization}

Table 2 reports the range for each operating parameter, the experimental analysis accounted for. Operation at both design point and under partial load underwent an in-depth investigation: the breakthrough of the plant overall performance was possible by a close-up analysis of the energy performance of each component, particularly the expander and the pump.

Table 2. Experimental campaign parameters and ranges of variation.

\begin{tabular}{ccc}
\hline Variable & Range & Units of Measure \\
\hline Mass flowrate - Water (evaporator) & $0,1-0,4$ & $\mathrm{~kg} / \mathrm{s}$ \\
Mass flowrate - Water (condenser) & $0,2-0,7$ & $\mathrm{~kg} / \mathrm{s}$ \\
Mass flowrate - R245fa & $35-75$ & $\mathrm{~g} / \mathrm{s}$ \\
Temperature - Water (evaporator inlet) & $80-112$ & ${ }^{\circ} \mathrm{C}$ \\
Temperature - Water (condenser inlet) & $15-22$ & ${ }^{\circ} \mathrm{C}$ \\
Upper cycle pressure & $6,1-11,9$ & $\mathrm{bar}$ \\
Lower cycle pressure & $0,6-3,5$ & $\mathrm{bar}$ \\
Mechanical power - expander & $200-750$ & $\mathrm{~W}$ \\
Torque - expander & $0,2-5,6$ & $\mathrm{Nm}$ \\
Mechanical power - pump & $150-280$ & $\mathrm{~W}$ \\
ORC-unit efficiency & $0,1-3,2$ & ${ }^{\circ}$ \\
\hline
\end{tabular}

To the scopes of the experimental characterization, a pair of $12 \mathrm{~kW}$ electric heaters provide thermal energy to the water inside the tank and mirror the variability of the solar source: the year-round available solar radiation at the experimental facility (town of L'Aquila, central Italy) on a 15 minutes time-basis is retrieved from [35] and the assessment of the thermal power to the storage unit refers to a $15 \mathrm{~m}^{2}$ solar thermal collector field, as presented in [34]. As the energy fed to the ORC-unit varies, a different mass- and energy-equilibrium is reached at the evaporator, leading to a different value of the processed mass flowrate of working fluid: a wide range $(35 \mathrm{~g} / \mathrm{s}-75 \mathrm{~g} / \mathrm{s})$ is considered for the prototype at hand. Each mass flowrate calls for a different value of expander RPM to maximize energy generation, particularly with volumetric expanders. In the case at hand, an additional factor to account for is the high sensitivity of sliding vane machines to the expansion ratio $\beta$ between the expander intake and exhaust pressures. 
Generally speaking, as the expander chamber approaches the discharge port, an imperfect matching between the chamber pressure and the exhaust one leads to two alternative situations:

1. If the chamber pressure is higher than the exhaust pressure an isochoric expansion takes place leading to a underexpansion phenomenon;

2. If the chamber pressure is higher than the exhaust one an isochoric compression occurs, resulting in an improper emptying, with a fraction of the mass flowrate trapped inside the vane and undergoing unwanted compression. Internal leaks (i.e., vane-to-vane transfer of working fluid under pressure gradients inside the expander) add further complexity to the problem of proper expansion modeling.

The cycle-by-cycle propagation of such a deviation from the ideality, results in an expansion efficiency that deteriorates quickly and eventually leads to poor energy performance of the cycle. In order to prevent under-expansion and over-compression from happening, the control of upper and lower cycle pressures is of the essence: the expansion ratio (i.e., expander intake-to-exhaust pressure ratio) should be kept as close as possible to the design value.

It is worth observing, though, that even if such detrimental effects associated with improper line-expander interaction were removed, the plant would perceive the expander as a concentrated pressure loss anyway. Indeed, the intake and discharge ports are not simultaneously open and during the filling phase, the expander acts as a closed valve that only opens once the expansion is complete. Between the intake port closing and the exhaust port opening, whereas the pressure upstream the expander increases (the expander is perceived as an obstruction to the fluid flow), the one downstream the expander decreases, due to insufficient fluid adduction. This is usually referred to as expander permeability [33]: littleto-no relief comes from the intake-to-exhaust leaks, i.e., from the mass of working fluid that runs from intake to exhaust through clearances, without providing any useful expansion work.

As a matter of fact, once one among the thermodynamic conditions (cycle pressures), the processed mass flowrate and the expander speed is assigned, the remaining variables are univocally fixed by the need for minimizing pressure disturbances and keeping the plant as close as possible to the design point.

\section{Results and Discussion}

Figure 2 accounts for the mass flowrate of working fluid as the independent variable, with the expander speed as a parameter: for any given mass flowrate, a change in the expander RPM leads to a different expansion ratio (Figure 2a), confirming the theoretical expectations on the expander permeability. As the revolution speed decreases, the permeability diminishes thus ensuring a higher expansion ratio that keeps the mass flowrate constant. On the contrary, the expander permeability raises with the revolution speed leading to a contraction of the expansion ratio for a certain mass flow rate provided by the pump. As a matter of fact, due to its tight relationship with the plant hydraulic permeability, the expander revolution speed can be considered as a variable for effective plant operation control: suitable operating points - in terms of mass flowrate and expansion ratio - span in a wide range ideally corresponding to the rectangular perimeter in Figure 2a.

Same goes for the expansion power, whose values $(250 \mathrm{~W}-750 \mathrm{~W})$ are even more dispersed, especially if compared to the pump power absorption, that shows a linear increase with the mass flowrate (Figure $2 \mathrm{~b}$ ), in the $200-280 \mathrm{~W}$ range. Figure $2 \mathrm{c}$ provides a clear indication that a maximum $500 \mathrm{~W}$ net power can be generated, with $62 \mathrm{~g} / \mathrm{s}$ of R245fa and 1250 RPM at the expander to which a $4 \%$ unit efficiency corresponds (Figure $2 \mathrm{~d}$ ). The unit efficiency is the ratio between the ORC net power and the thermal power fed to R245fa (eq.1). The ORC net power is the difference between the power generated at the expander and the pump power absorption.

$$
\eta_{O R C}=\frac{P_{O R C}}{P_{t h}}=\frac{P_{e x p}-P_{p m p}}{P_{t h}}
$$

Therefore, despite it is conceived as a stationary application, the ORC unit is fit to work in a wide operating range and still assure satisfying levels of power and plant efficiency, mainly thanks to the performance robustness of volumetric machines to both R245fa mass flowrate variability and working fluid condition at expander inlet (Figure 2). When the plant works at its design point $-62 \mathrm{~g} / \mathrm{s} \mathrm{R} 245 \mathrm{fa}, \beta=7$ and $1250 \mathrm{RPM}$ expander speed - it achieves the maximum power and efficiency. A further degree of flexibility comes from the possibility to vary the expander and pump speed, and to reach the desired operating point in terms of mass flow rate and expansion ratio, thus allowing a proper plant performance even in presence of a thermal power below the design value. 


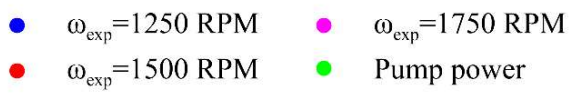

(a)

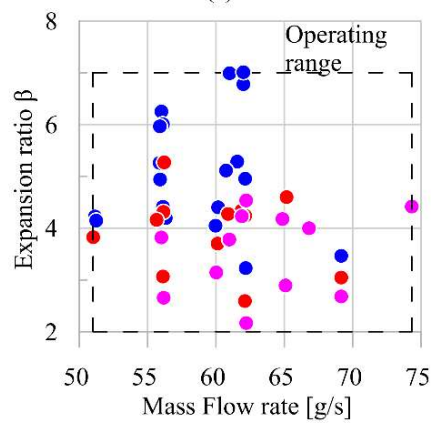

(c)

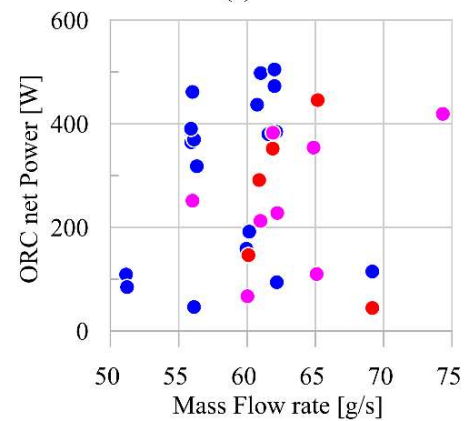

(b)

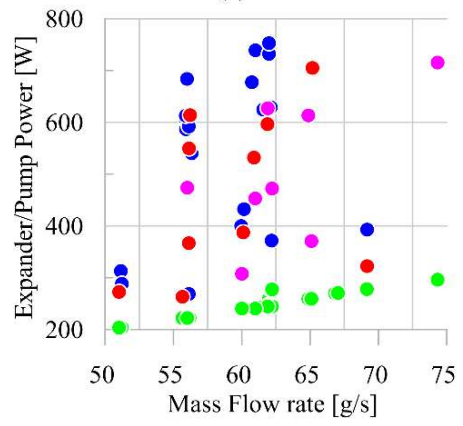

(d)

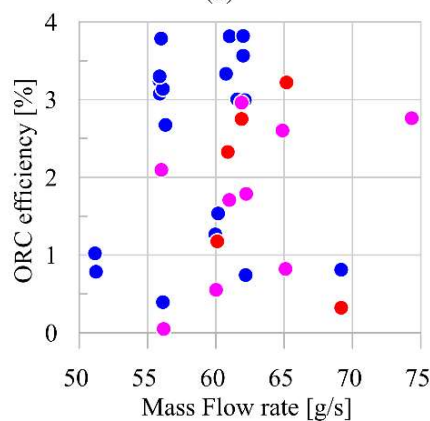

Fig. 2. Experimental results - Expansion ratio (a), Expander/Pump power (b), ORC net power (c) and efficiency (d) as funcion of mass flow rate.

$\bullet \omega_{\mathrm{exp}}=1250 \mathrm{RPM} \bullet \omega_{\mathrm{exp}}=1500 \mathrm{RPM} \quad \bullet \quad \omega_{\exp }=1750 \mathrm{RPM}$

(a)

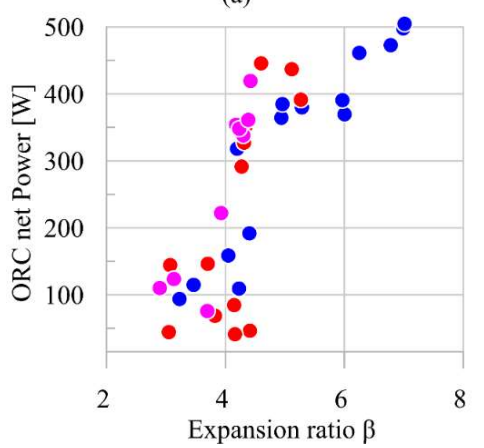

Fig. 3. Experimental results - ORC-plant net power (a) and efficiency (b) as fucntion of the expansion ratio. (b)

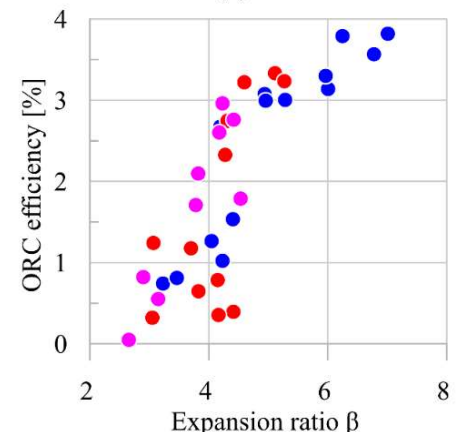


The ORC net power and efficiency are reported also as function of expansion ratio in Figure 3a and 3b, respectively: they both increase as the expansion ratio grows., i.e., as a larger enthalpy difference is exploited through the expansion.

Moreover, also the impact of pump power on the expander one diminishes as expansion ratio raises as it can be seen observing the Back-Work ratio reported in Figure 4. The Back-Work ratio BWR is defined as in eq.2:

$$
B W R=\frac{P_{p m p}}{P_{e x p}}
$$

where $P_{p m p}$ and $P_{\exp }$ are the power at the pump and the expander, respectively. Figure 4 shows that BWR quite follows a hyperbolic decrease when the expansion ratio grows from 3 to 7 : whereas at design point the expansion ratio is $27 \%$, when the expansion ratio equals 3 , the expander power is almost entirely eroded by the pump one, with obvious penalty on the net ORC power.

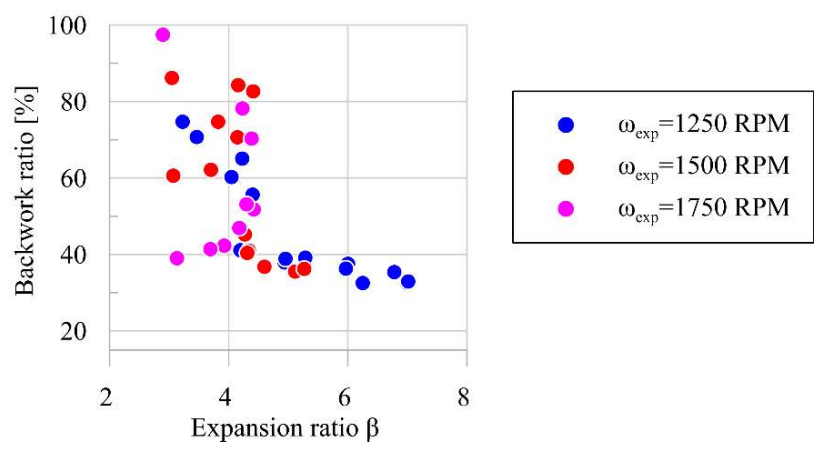

Fig. 4. Back-Work ratio as function of expansion ratio.

As commonly retained in literature, the expander is the key component of an ORC-based power unit, for its efficiency highly affects both the attainable plant power and efficiency. Characteristic values of the expander generated power and efficiency for the case at hand are in Figures 5a and 5b, respectively: whereas a linear growth with the expansion ratio is appreciated at 1250 RPM expander speed, a less defined trend is detected with different expander speeds.

Such experimental evidence suggests that the beneficial effect of a higher expander speed compensates the reduction of the expansion ratio due to the permeability increase, to keep the mass flowrate constant. The maximum expander power is available at design point $\left(\beta=7, \omega_{\exp }=1250 \mathrm{RPM}\right)$ - which matches with the previously observed trend - as well as at points with $\beta=3, \omega_{\exp }=1500$ RPM and $\omega_{\exp }=1750$ RPM: the higher BWR, though, results in a lower ORC net power. 


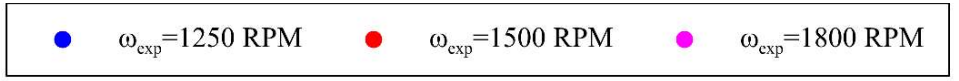

(a)

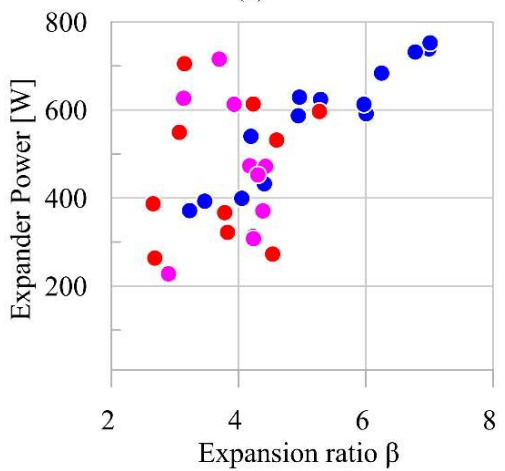

(b)

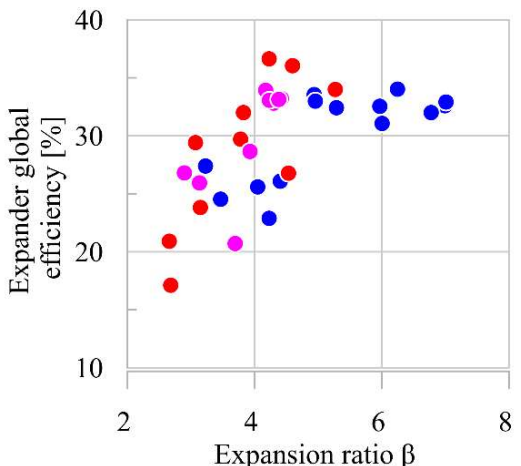

Fig. 5. Experimental results - Expander global (a) and volumetric efficiency (b) as function of expansion ratio.

\section{Conclusions}

A thorough experimental campaign, carried out on a prototype ORC-unit, allowed the characterization of a smallscale (below $1 \mathrm{kWe}$ ) recovery plant for solar micro-cogeneration in the residential sector. The main interest for the unit, featuring standard on-the-shelf components and R $245 \mathrm{fa}$ as the working fluid, comes from the assessment of the feasibility of simultaneous heat recovery and electricity generation from very low-grade thermal energy (below $110^{\circ} \mathrm{C}$ ), characterized by intermittent availability, due to seasonal fluctuations and withdrawals for DHW production.

This calls for a plant able to perform under off-design conditions, with little energy penalty, and, eventually, for a dedicated control strategy, that allows a convenient trade-off between power generation and coverage of the thermal demand for domestic purposes. In order for the prototype to reproduce real operating conditions, the testing activity accounted for different sets of operating parameters - namely, threshold temperature for ORC-unit activation, cycle pressures, expander speed, pump speed - and led to the mapping of multiple operating points.

On the one hand, the available thermal power at the evaporator fixes the equilibrium mass flowrate of working fluid, which in turn fixes the pump speed. On the expander side, the permeability increases with the expander speed, leading to lower losses for the unit and a lower overpressure for the pump to overcome.

If the expander speed is constrained, any variation in the available thermal power induces off-design operation and low energy output. An effective option to tackle such issue is to define a control strategy accounting for independent speed control for the expander and the pump. By decoupling the two speed controls, a twofold advantage is attained: the pump adapts the processed mass flowrate to the actual thermal availability and assures the maximum heat recovery from the upper thermal source; the expander speed can be adjusted to variable thermodynamic conditions (cycle pressures and superheating degree) and pursue the optimum trade-off between enhanced lubrication, optimum volumetric performance, and high permeability. The experimental results shown the capability of the whole system to work in off design condition. At design point (expansion ratio equal to 7) the efficiency is equal to $4 \%$ and the produced net power is $500 \mathrm{~W}$. However, even if the operating condition are far from the rated one, the plant allows to achieve satisfying power $(300 \mathrm{~W}-400 \mathrm{~W})$ and efficiency $(2 \%-3 \%)$, with expansion ratio equal to 4 and 6 .

The experimental characterization shows that the expander performance highly affects the whole plant efficiency and produced power. Moreover, the results shows that the machine presents large room of improvement on the volumetric efficiency, currently not exceeding 55\%. A better understanding of the oil distribution inside the machine and the definition of the optimum amount of oil for mixing with working fluid will play a fundamental role in this sense. The fact that the maximum efficiency is not achieved at the design point, but when the expander rotates at 1500 RPM and the pressure ratio is around 4 , with an expander efficiency close to $40 \%$, invites to reconsider the role of the volumetric efficiency in energy performance of volumetric machines for small scale applications and to design proper experimental activity to assess it, during normal operation.

\section{Acknowledgements}

This work has been developed in collaboration with Sivam S.r.l. Mr. Paolo Orsini, President of the Company, is particularly acknowledged for technical and financial support and Mr. Alberto Zurlino for test rig development. 


\section{Nomenclature}

\begin{tabular}{cccc}
\hline \multicolumn{4}{c}{ Symbols } \\
\hline$P$ & Power [W] & $\eta$ & Efficiency [\%] \\
\hline$p$ & Pressure [bar] & $\beta$ & Pressure ratio [-] \\
\hline$\dot{m}$ & Mass flowrate [kg/s] & $\omega$ & Revolution speed \\
\hline$\dot{W}$ & Power [W] & $\beta$ & Expansion ratio \\
\hline
\end{tabular}

\begin{tabular}{cccc}
\hline \multicolumn{4}{c}{ Subscripts } \\
\hline ev & evaporator & exp & expander \\
\hline wf & working fluid & v & volumetric \\
\hline net & net & pmp & pump \\
\hline orc & Organic Rankine Cycle & th & thermal \\
\hline
\end{tabular}

\section{References}

1. J. S. Pereira, J. B. Ribeiro, R. Mendes, J. C. André, “Analysis of a hybrid (topping/bottoming) ORC based CHP configuration integrating a new evaporator design concept for residential applications", Applied Thermal Engineering, 160, 1-11, (2019).

2. D. Mikielewicz, J. Mikielewicz, "A thermodynamic criterion for selection of working fluid for subcritical and supercritical domestic micro-CHP”, Applied Thermal Engineering, 30, 2357-2362, (2010).

3. A. Franco, F. Bellina, "Methods for optimized design and management of CHP systems for district heating networks (DHN)", Energy Conversion and Management, 172, 21-31, (2018).

4. L. Cioccolanti, R. Tascioni, A. Arteconi, "Simulation analysis of an innovative micro-solar $2 \mathrm{kWe}$ Organic Rankine Cycle plant for residential applications", Energy Procedia, 142, 1629-1634, (2017).

5. A. M. Pantaleo, S. M. Camporeale, A. Miliozzi, V. Russo, N. Shah, C. N. Markides, "Novel hybrid CSP-biomass CHP for flexible generation: Thermo-economic analysis and profitability assessment", Applied Energy, 204, 994-1006, (2017).

6. J. Wang, Z. Yan, P. Zhao, Y. Dai, "Off-design performance analysis of a solar-powered organic Rankine cycle", Energy Conversion and Management, 80, 150-157, (2014).

7. P. Ouyang, Y. Xu, L. Qi, S. Xing, H. Fooladi, "Comprehensive evaluation of flat plate and parabolic dish solar collectors' performance using different operating fluids and MWCNT nanofluid in different climatic conditions", Energy Reports, 7, 24362451, (2021).

8. M. Petrollese, D. Cocco, "Robust optimization for the preliminary design of solar organic Rankine cycle (ORC) systems", Energy Conversion and Management, 184, 338-349, (2019).

9. CODE 2 - Cogeneration Observatory and Dissemination Europe. Micro-CHP potential analysis - European level report, (2014).

10. M. Astolfi, "Techno-economic Optimization of Low Temperature CSP Systems Based on ORC with Screw Expanders", Energy Procedia, 69, 1100-1112, (2015).

11. M. Peters, T. S. Schmidt, D. Wiederkehr, M. Schneider, "Shedding light on solar technologies-A techno-economic assessment and its policy implications", Energy Policy, 39, 6422-6439, (2011).

12. J. M. Rodríguez, D. Sánchez, G. S. Martínez, E. Bennouna, B. Ikken, "Techno-economic assessment of thermal energy storage solutions for a 1MWe CSP-ORC power plant", Solar Energy, 140, 206-218, (2016).

13. W. Fan, Z. Han, P. Li, Y. Jia, "Analysis of the thermodynamic performance of the organic Rankine cycle (ORC) based on the characteristic parameters of the working fluid and criterion for working fluid selection", Energy Conversion and Management, 211, 1-18, (2020).

14. S. Quoilin, M. Orosz, H. Hemond, V. Lemort, "Performance and design optimization of a low-cost solar organic Rankine cycle for remote power generation", Solar Energy, 85, 955-966, (2011).

15. R. Kong, T. Deethayat, A. Asanakham, N. Vorayos, T. Kiatsiriroat, "Thermodynamic performance analysis of a R245fa organic Rankine cycle (ORC) with different kinds of heat sources at evaporator", Case Studies in Thermal Engineering, 13, 1-10, (2019).

16. M. Santos, J. André, E. Costa, R. Mendes, J. Ribeiro, "Design strategy for component and working fluid selection in a domestic micro-CHP ORC boiler", Applied Thermal Engineering, 169, (2020).

17. J. Lizana, C. Bordin, T. Rajabloo, "Integration of solar latent heat storage towards optimal small-scale combined heat and power generation by Organic Rankine Cycle", Journal of Energy Storage, 29, (2020).

18. A. M. Pantaleo, S. M. Camporeale, A. Sorrentino, A. Miliozzi, N. Shah, C. N. Markides, "Solar/biomass hybrid cycles with thermal storage and bottoming ORC: System integration and economic analysis", Energy Procedia, 129, 724-731, (2017).

19. D. Vittorini, A. Antonini, R. Cipollone, R. Carapellucci, C. Villante, "Solar Thermal-Based ORC Power Plant for Micro Cogeneration - Performance Analysis and Control Strategy”, Energy Procedia, 148, (2018). 
20. P. K. S. Rathore, S. K. Shukla, "Enhanced thermophysical properties of organic PCM through shape stabilization for thermal energy storage in buildings: A state of the art review", Energy and Buildings, 236, 1-29, (2021).

21. C. Prieto, L. F. Cabeza, "Thermal energy storage (TES) with phase change materials (PCM) in solar power plants (CSP). Concept and plant performance", Applied Energy, 254, (2019).

22. S. H. Gage, D. Kesseli, J. Dupree, C. Kimbal, J. Rigby, J. Yates, B. Morrison, G. Bigham, C. S. Turchi, "Technical and economic feasibility of molten chloride salt thermal energy storage systems", Solar Energy Materials and Solar Cells, 226, 1-8, (2021).

23. X. Li, E. Xu, S. Song, X. Wang, G. Yuan, "Dynamic simulation of two-tank indirect thermal energy storage system with molten salt, Renewable Energy", 113, 1311-1319, (2017).

24. W. Wang, S. Guo, H. Li, J. Yan, J. Zhao, X. Li, J. Ding, "Experimental study on the direct/indirect contact energy storage container in mobilized thermal energy system (M-TES)", Applied Energy, 119, (2014).

25. J. Xu, C. Yu, "Critical temperature criterion for selection of working fluids for subcritical pressure Organic Rankine cycles", Energy, 74, 719-733, (2014).

26. K. Thurairaja, A. Wijewardane, S. Jayasekara, C. Ranasinghe, "Working Fluid Selection and Performance Evaluation of ORC", Energy Procedia, 156, 244-248, (2019).

27. D. Vittorini, R. Cipollone, R. Carapellucci, "Enhanced heat exchanger layout for optimum energy performance in solar thermal ORC-based unit", AIP Conference Proceedings, 2191, (2019).

28. D. Vittorini, R. Cipollone, R. Carapellucci, "Enhanced performances of ORC-based units for low grade waste heat recovery via evaporator layout optimization”, Energy Conversion and Management, 197, (2019).

29. M. Wang, Y. Chen, Q. Liu, Z. Yuanyuan, "Thermodynamic and thermo-economic analysis of dual-pressure and single pressure evaporation organic Rankine cycles", Energy Conversion and Management, 177, 718-736, (2018).

30. T. Li, Z. Zhang, J. Lu, J. Yang, Y. Hu, "Two-stage evaporation strategy to improve system performance for organic Rankine cycle", Applied Energy, 150, 323-334, (2015).

31. M. Imran, M. Usman, B. Park, D. Lee, "Volumetric expanders for low grade heat and waste heat recovery applications", Renewable and Sustainable Energy Reviews, 57, 1090-1109, (2016).

32. P. Song, M. Wei, L. Shi, S. N. Danish, C. Ma, "A review of scroll expanders for organic Rankine cycle systems", Applied Thermal Engineering, 75, 54-64, (2015).

33. F. Fatigati, M. Di Bartolomeo, D. Di Battista, R. Cipollone, “A dual-intake-port technology as a design option for a Sliding Vane Rotary Expander of small-scale ORC-based power units", Energy Conversion and Management, 209, (2020).

34. D. Vittorini, A. Antonini, R. Cipollone, R. Carapellucci, "Multi-Variable Control and Optimization Strategy for Domestic SolarORC Combined Heat and Power Generation System", E3S Web Conf., 197, (2020).

35. https://midedmz.nrel.gov/solpos/solpos.html 